\title{
Terahertz generation from single and multiple filaments in air
}

\author{
O. Kosareva ${ }^{1,2,3}$, N. Panov ${ }^{1,2}$, D. Shipilo ${ }^{1,2}$, V. Andreeva ${ }^{1,4}$, T.-J. Wang ${ }^{5}$, Y. Chen ${ }^{6}$, \\ W. Liu ${ }^{3}$, A. Savel'ev ${ }^{1}$, and A. Shkurinov ${ }^{1}$ \\ ${ }^{1}$ Lomonosov Moscow State University, 1/2 Leninskie gori, Moscow, Russia, kosareva@ physics.msu.ru \\ ${ }^{2}$ Lebedev Physical Institute of Russian Academy of Sciences, Moscow, Russia \\ ${ }^{3}$ Institute of Modern Optics, Nankai University, Tianjin, China \\ ${ }^{4}$ School of Mathematics, University of Minnesota, Minneapolis, Minnesota, USA \\ ${ }^{5}$ Shanghai Institute of Optics and Fine Mechanics, Chinese Academy of Sciences, Shanghai, China \\ ${ }^{6}$ Shanghai Jiao Tong University, Shanghai, China
}

Terahertz $(\mathrm{THz})$ photonics is entering an era of strong electromagnetic fields in the $\mathrm{THz}$ frequency range [1]. Optical breakdown plasma takes a specific place among $\mathrm{THz}$ sources. With the appearance of compact commercial terawatt laser systems and the actualization of remote sensing tasks, two-color optical breakdown in air has turned into a powerful mechanism of broadband $\mathrm{THz}$ generation at the observation point [2]. There are several problems associated with $\mathrm{THz}$ remote sensing applications. First, the directional diagram of emitted $\mathrm{THz}$ radiation usually has a conical structure [3]. Nevertheless, as the filament length increases, the divergence angle decreases [4]. Second, the limitation of air-breakdown $\mathrm{THz}$ applications to remote-sensing tasks is due to low signal-to-noise ratio in the amplitude analysis of $\mathrm{THz}$ radiation reflected from the object under study. Therefore, one can use polarization analysis of useful signals whose effective application requires thorough control of the state of polarization of $\mathrm{THz}$ radiation. Third, further optimization of the $\mathrm{THz}$ yield from two-color filaments is required to approach the signal level obtained from optical rectification in crystals [1]. Control over the relative temporal (and spatial) properties of the optical pump fields, e.g. via the pulse chirp, offers an important means to control and optimize the optical-to- $\mathrm{THz}$ conversion process. With increasing pulse peak power and transition to multiple filamentation more overall $\mathrm{THz}$ energy is generated however the proper regularization of the high-peak-power beam is needed to provide for the maximum $\mathrm{THz}$ yield in the narrow angle.

In this paper we present a series of studies for the control and optimization of $\mathrm{THz}$ directionality, polarization and energy yield.

We observe linear-to-elliptical transformation of $\mathrm{THz}$ radiation through independent control of initially linear polarization directions of $\omega$ and $2 \omega$ beams. Strongly elliptical $\mathrm{THz}$ radiation appears abruptly at the threshold angle of $\sim 85^{\circ}$ between the $\omega$ and $2 \omega$ light fields. The physical reason for $\mathrm{THz}$ beam ellipticity was identified by us as the nonlinear response of the birefringent medium induced by an $800 \mathrm{~nm}$ pump. We found $\mathrm{THz}$ ellipticity and energy yield using a vectorial nonparaxial propagation equation (Fig. 1).

We have demonstrated and justified the mechanism which dictates the optimum chirp to maximize the $\mathrm{THz}$ yield from a two-color filament. We have experimentally measured the $\mathrm{THz}$ energy yield using 800-nm pulses in a single filament regime, being chirped from 35 fs up to $250 \mathrm{fs}$ both positively and negatively. We found that the positively chirped pulse proves to be a more advantageous choice since the $\omega-$ $2 \omega$ pulse overlap is larger by $\sim 15$ fs as compared with the transform-limited pulse (Fig. 2).

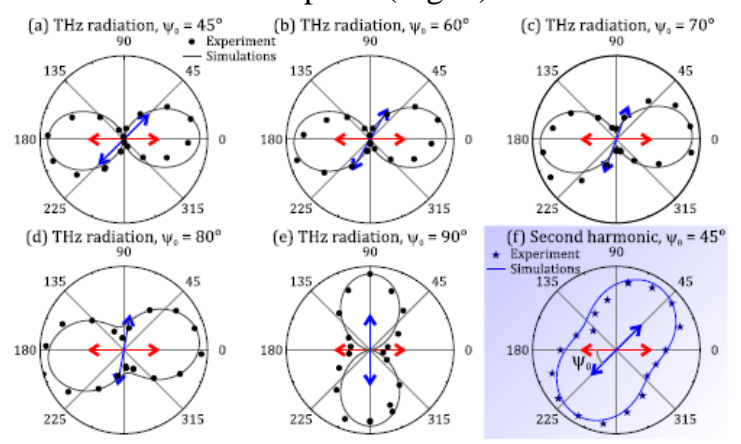

Fig. 1 (a)-(e) THz energy after transmission through $\mathrm{THz}$ analyzer, in dependence on its rotation angle, for different angles $\psi_{0}$, for different angles $\psi_{0}$ between polarization directions of the $800 \mathrm{~nm}(\omega)$ and $400 \mathrm{~nm}(2 \omega)$ beams. (f) Second-harmonic energy after its transmission through polarization analyzer. Red (blue) arrow indicates the incident polarization of the $\omega(2 \omega)$ beam.

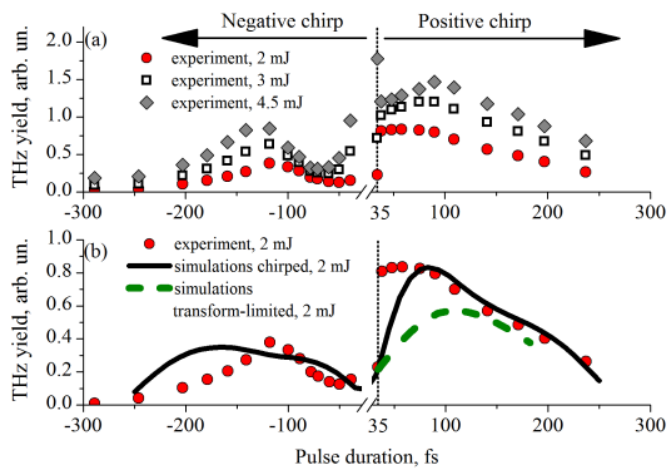

Fig. 2 (a) The experimentally measured dependence of the THz energy on the pump $(800 \mathrm{~nm})$ chirped pulse duration. The pump energy is fixed for each chirped pulse duration variation in the range from -300 fs (FWHM, negatively chirped) to +250 fs (FWHM, positively chirped). (b) The experimental vs. simulated dependence of the THz energy on the 800-nm 2-mJ chirped pulse duration. Green dashed curve shows simulated $\mathrm{THz}$ yield for the transform-limited pulse.

We have shown that a $2 D$ array of multiple plasma filaments in atmospheric density gases leads to the efficient increase of terahertz radiation emitted in the forward direction. As the number of filaments in an $N \times N$ array is augmented, the $\mathrm{THz}$ energy increase per unit polar angle in the central cone exceeds the standard gain factor $N^{2}[4,5]$. The two-dimensional multiple filament array can be produced from an actu- 
al non-ideal elliptical beam using a phase plate to introduce a phase barrier and block the interaction between the neighboring filaments. Such regularized structure of plasma channels is used to control the directional diagram of $\mathrm{THz}$ radiation from a subterawatt-peak-power $800 \mathrm{~nm}+400 \mathrm{~nm}$ femtosecond laser pulse.
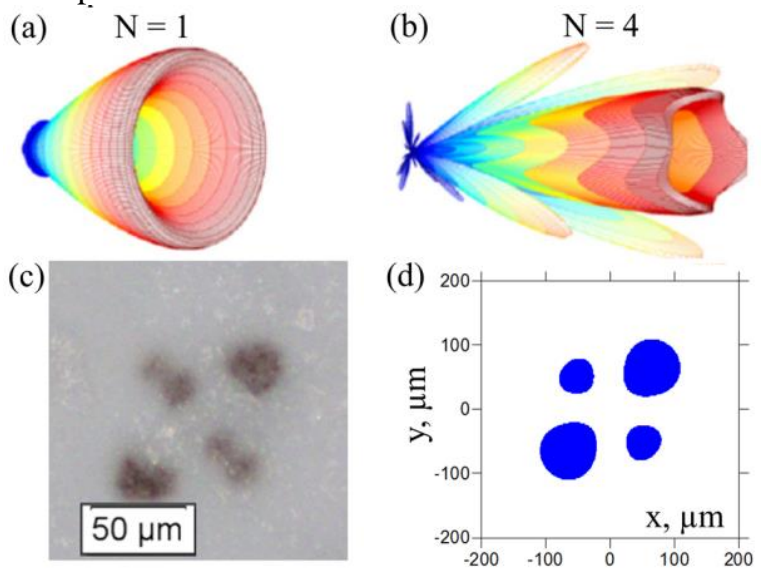

Fig. 3. (a, b) Full 3D angular $\mathrm{THz}$ intensity distribution from an array of $N$ multiple filaments with the length $L=1 \mathrm{~cm}$. (c) Single-laser-shot burnt photo paper images after the geometrical focus of 1.1-mJ 35-fs $800 \mathrm{~nm}+400$ $\mathrm{nm}$ elliptical beam transported through a phase mask in air. The reproduction of the plasma channels in $3 D+$ time carrier resolved simulations is given in (d).

This work is financially supported by Russian Science Foundation (RSF, Grant No. 16-42-01060), the National key research and development program (2018YFB0504400), "111 Project (B16027)" the National Basic Research Program of China
(2014CB339801); the National Natural Science Foundation of China (11474202, 11774228, 11721091); the Strategic Priority Research Program of the Chinese Academy of Sciences (XDB16000000); Key Project from Bureau of International Cooperation Chinese Academy of Sciences (181231KYSB20160045).

\section{References}

1. X.-C. Zhang, A. Shkurinov, and Y. Zhang. Extreme terahertz science // Nat. Photonics 2017. V. 11, p. 16.

2. T.-J. Wang, S. Yuan, Y. Chen, J.-F. Daigle, C. Marceau, F. Théberge, M. Châteauneuf, J. Dubois, and S. L. Chin. Toward remote high energy terahertz generation // Appl. Phys. Lett. 2010. V. 97, p. 111108.

3. V. Andreeva, O. Kosareva, N. Panov, D. Shipilo, P. Solyankin, M. Esaulkov, P. G. de Alaiza Martínez, A. Shkurinov, V. Makarov, L. Bergé, and S. L. Chin. Ultrabroad terahertz spectrum generation from an air-based filament plasma // Phys. Rev. Lett. 116, 063902 (2016).

4. N. Panov, V. Andreeva, O. Kosareva, A. Shkurinov, V. A. Makarov, L. Bergé and S. L. Chin. Directionality of terahertz radiation emitted from an array of femtosecond filaments in gases // Laser Phys. Lett. 2014. V. 11, p. 125401.

5. S. I. Mitryukovskiy, Y. Liu, B. Prade, A. Houard, and A. Mysyrowicz. Coherent synthesis of terahertz radiation from femtosecond laser filaments in air // Appl. Phys. Lett. 2013. V. 102, p. 221107. 